\title{
The use of the laryngeal tube disposable by paramedics during out-of-hospital cardiac arrest: a prospectively observational study (2008-2012)
}

\author{
Jens-Uwe Müller, ${ }^{1}$ Thomas Semmel, ${ }^{2}$ Roland Stepan, ${ }^{2}$ Timo F Seyfried, ${ }^{1}$ \\ Aron F Popov, ${ }^{3}$ Bernhard M Graf, ${ }^{1}$ Christoph H R Wiese ${ }^{1}$
}

\begin{abstract}
${ }^{1}$ Department of Anaesthesiology, University Medical Centre Regensburg, Regensburg, Germany

${ }^{2}$ Medical administrative district of Fulda, Fulda, Germany ${ }^{3}$ Department of Cardiothoracic Surgery, University Medical Centre Goettingen, Göttingen, Germany
\end{abstract}

Correspondence to PD Dr Christoph H R Wiese, Department of Anaesthesiology, University Medical Centre Regensburg, Franz-Josef-Strauß-Allee 11, Regensburg D-93053, Germany;

christoph.wiese@ukr.de

Received 29 August 2012 Revised 22 October 2012 Accepted 25 October 2012

Published Online First

10 January 2013

\footnotetext{
To cite: Müller J-U, Semmel T, Stepan R, et al. Emerg Med $\mathrm{J}$

2013;30:1012-1016.
}

\begin{abstract}
Summary In the previous and the current guidelines of the European Resuscitation Council (ERC), endotracheal intubation (ETI), as an instrument for ventilation during resuscitation, was confirmed as less important for paramedics not trained in this method. For those, during resuscitation, the laryngeal tube is recommended by the ERC as a supraglottic airway device. The present study investigated prospectively the use of the laryngeal tube disposable (LT-D) by paramedics in prehospital emergency cases.
\end{abstract}

Methods During a 42-month period (Sept 2008-Feb 2012), we prospectively registered all prehospital cardiac arrest situations in which the LT-D had been applied by paramedics (from one emergency medical service in Germany).

Results During the defined period, 133 attempts, recorded on standardised data sheets, were enrolled into the investigation. Three were excluded from the study because of use during a trauma situation. Therefore, 130 patients were evaluated in this study. For this, the LT-D was used in $98 \%$ of all cases during resuscitation, and in about $2 \%$ of other emergencies (eg, trauma). With regard to resuscitation, adequate ventilation/oxygenation was described as possible in $83 \%$ of all included cases. In $66 \%$ of all cases, no problems concerning the insertion of the LT-D were described by the paramedics. No significant problems were reported in $93 \%$. In $7 \%$ ( $n=9$ cases), no insertion of the LT-D was possible. Instead of bag-mask-valve ventilation, the LT-D was used as a first-line airway device in about $66 \%$. Between the two defined groups, no statistically significant differences were found ( $p>0.05$ ).

Conclusions As an alternative airway device during resuscitation, recommended by the ERC in 2005 and 2010, the LT-D may enable ventilation rapidly and, as in most of our described cases, effectively. Additionally, by using the LT-D in a case of cardiac arrest, a reduced 'hands-off time' and, therefore, a high chest compression rate may be possible. Our investigation showed that the LT-D was often used as an alternative to bag-mask-ventilation and to ETI as well. However, we were able to describe more problems in the use of the LT-D than earlier investigations. Therefore, in future, more studies concerning the use of alternative airway devices in comparison with ETI and/or videolaryngoscopy seem to be necessary.

\section{INTRODUCTION}

In November 2005 and 2010, the European Resuscitation Council (ERC) released their revised versions of the resuscitation guidelines for Advanced
Life Support (ALS) in which the following two adjustments were incorporated as very important: ${ }^{12}$

\section{Emphasis on chest compression}

Successful management of a patient during cardiac arrest must include high-quality chest compressions (100-120 per minute) and a 'hands-off time' (HOT; time during which there are no chest compressions) that is as short as possible. ${ }^{13}$ These facts are important because nearly continuous chest compressions are required to maintain tissue perfusion. ${ }^{3}$

\section{Expansion of airway management options}

The gold standard for securing the airway is considered to be endotracheal intubation (ETI). ETI should only be attempted by trained healthcare providers having adequate ongoing experience with this technique; because of airway management, thoracic compressions should not be paused for more than $10 \mathrm{~s}^{1}{ }^{1}$ Because of the high incidence of complications (eg, oesophageal intubation), personnel without experience in ETI should use alternative airway devices (eg, laryngeal mask, laryngeal tube) to manage the airway during cardiac arrest. ${ }^{12}$ If it is not possible to insert alternative airway devices as soon as possible, paramedics may resort to bag-mask-valve (BMV) ventilation. ${ }^{1}$

One of the supraglottic airway devices (SADs), the laryngeal tube disposable (LT-D), was used in the present investigation. The laryngeal tube was introduced in 1999 as an alternative device for protecting the difficult airway. ${ }^{4-7}$ Because of easy insertion/handling and a reported insertion success rate of about $92-100 \%$, the LT-D seems to be an adequate alternative airway device during resuscitation and for the management of difficult airways. 135 8 3 -12

In 2006, the LT-D was introduced as an experimental SAD for paramedics in the investigated region. Because of the high incidence of complications and the poor success rate of ETI performed by paramedics, and because of the ERC guidelines in 2005 and 2010, in our investigated 'emergency medical service' (EMS) the LT-D has become paramedics' standard way of managing the airway during resuscitation since $2008 .^{12^{8-10}}$ The LT-D is designed for blind insertion without laryngoscopy. ${ }^{11}$ It has undergone laboratory and practical evaluation concerning alternative airway management during cardiac arrest, especially for rescuers who are not experienced in ETI. ${ }^{8} 10$

In the present investigation, we have evaluated the experiences of paramedics after using the LT-D 
to manage the airway during resuscitation. Therefore, we evaluated the time taken for insertion of the LT-D, the reason for its use, the problems and complications during placement/insertion/ventilation, and the return of spontaneous circulation (ROSC) during resuscitation.

\section{METHODS}

Over the course of 42 months, 130/133 standardised records from one EMS system (City of Fulda, Germany) were integrated into this investigation. The study design was an observational one. All prehospital uses of the LT-D performed by paramedics were recorded by means of a standardised sheet. Based on the emergency documents and the observational sheet, we only evaluated the use of the LT-D (Size 3-5; VBM Medizintechnik $\mathrm{GmbH}$, Sulz a.N., Germany) as an alternative SAD during resuscitation.

Due to the high incidence of complications using ETI and the high HOTs during resuscitation, we decided to attempt the use of an alternative SAD for paramedics from our EMS in 2006. Since 2008 (according to the new ERC ALS guidelines in 2005 and good experiences during the experimental phase), the LT-D is now the standard SAD for cardiac arrest situations which are treated by paramedics. Prior to using the LT-D as an alternative device, a 90 min airway training session, according to the manufacturer's instructions, had to be completed by every paramedic in the investigated emergency department. ${ }^{1}{ }^{13}$ Because paramedic education in Germany includes repetitive training in airway management with alternative airway devices, all paramedics were considered as experienced in airway management by using SADs. Furthermore, all paramedics from the investigated EMS have to repeat a 4-8 h 'ALS' course, according to the ERC guidelines, once every year. This course includes a 45-90 min airway training session concerning SADs and BMV.

Demographic patient data, such as age, gender, height and the size of the inserted laryngeal tube, were recorded prospectively. The specific research objectives and data obtained by a standardised study sheet were:

- Time required for LT-D placement $(<10 \mathrm{~s}, 10-20 \mathrm{~s}$, 21-30 s, >30 s).

- Each single attempt should be accomplished within $10-30 \mathrm{~s}$, as recommended by the ERC in 2005 and $2010 .^{1}{ }^{2}$ The patients had to be ventilated by using the LT-D. In case of failed insertion or inadequate oxygenation of the patient, the paramedics were instructed to use BMV ventilation.

- Number of attempts to correctly place the LT-D (free answers).

- Point in time of LT-D insertion during resuscitation (multiple-choice answers).

- Indication for LT-D placement (multiple-choice answers).

- Reasons for the replacement of the LT-D (multiple-choice answers).

- Successful ventilation after the LT-D was inserted (determined by pulmonary auscultation; multiple-choice answers).

- Oxygen rate before and after the LT-D was inserted (multiple-choice answers).

- Reasons for replacement of the LT-D (the LT-D was not left in place throughout resuscitation and transport; multiple-choice answers).

- Return of spontaneous circulation (ROSC) (yes/no answer).

- Complications/problems concerning the use of the LT-D during resuscitation (free answers).
The time taken for insertion of the LT-D was recorded by every paramedic/resuscitation team on his/their own (in most cases, a stopwatch was used). For this reason, we decided to use a time range and not the exact times taken for insertion. As per the definition of the study, timing was started after the paramedics had decided to use the LT-D as an alternative airway device. Until 2010, chest compressions were stopped while the LT-D was inserted; since 2011, chest compressions have not been stopped during the use of the LT-D. Time taken for placement was recorded by a paramedic who was not immediately involved in providing care. Time for placement was stopped at the beginning of the first successful ventilation after the LT-D had been inserted. Adequate ventilation was defined as when breath sounds could be heard over both lungs, and chest movement could be seen. After the LT-D had been inserted correctly, the paramedics performed continuous chest compressions. In line with Wik et al, we defined the HOT as time during which no chest compressions occur. ${ }^{14}$

Each ambulance was equipped with three sizes of the LT-D for adult patients (sizes 3, 4 and 5), and the paramedics were instructed to attempt to use the LT-D instead of ETI and/or BMV ventilation in all cases of cardiac arrest. ROSC was defined as the ROSC during resuscitation which made it possible to transport the patient to hospital without ongoing resuscitation. ROSC after $24 \mathrm{~h}$ was defined as spontaneous circulation during the next $24 \mathrm{~h}$ after resuscitation. Where possible, paramedics and the investigators tried to obtain this special information within $48 \mathrm{~h}$ after resuscitation. Following an emergency, the paramedics had to use the standardised study protocol which is based on the standardised emergency protocol and the participants' information. Only resuscitations of adult patients were included.

\section{Ethics, data extraction and statistical analysis}

In line with the ethical regulations of the Declaration of Helsinki, the data were collected anonymously so that no conclusions could be drawn on single patients or paramedics. ${ }^{15}$ The local ethical commission was informed about the study, and ethical clearance was obtained from the institute's ethics commission (University Medical Centre, University of Regensburg, Germany). Ethics committee approval was not necessary for the study because no clinical procedures were performed, and patient records were not used (ethical commission sign $\mathrm{JvH}$ 2/10/2011-1344).

With regard to statistical analysis, we defined the following investigation groups: ERC 2005 versus ERC 2010; working experience $<10$ years versus working experience $>10$ years. Study variables were expressed as median, range, total number and/or per cent as appropriate. Tabulation of the data was carried out in Excel 2010 (MS Excel, Microsoft Inc, 2010). For tabulation of the data and descriptive statistics, Excel 2010 was used, and statistical data analysis, where necessary, was performed with SPSS 18.0 (SPSS Inc, USA, 2010). A statistically significant difference between the defined groups was calculated with the Wilcoxon signed rank test and the analysis of variance for non-parametric data. A p value of 0.05 was considered to be statistically significant. Correction for multiple testing was performed according to Sidak. ${ }^{16}$

\section{RESULTS}

During the study period of 42 months (September 2008 to February 2012), there were 133 standardised records in which the LT-D was used by paramedics to manage the airway during resuscitation $(n=130)$ and trauma cases $(n=3)$. In the following, 
only the applications during resuscitation were displayed. In according with the ERC guidelines 2005, and with the ERC guidelines 2010, $\mathrm{n}=88 \quad(67.7 \%)$ and $\mathrm{n}=42 \quad(32.3 \%)$ uses, respectively, of the LT-D were documented. Overall, there were no statistically significant differences between the defined groups $(p>0.05)$. Therefore, data are presented for all cases.

Overall, about 75 resuscitation attempts, following out-of-hospital cardiac arrest, per year occurred in the investigated region (own data). Thus, the LT-D was used in about 50\% of all cardiac arrest cases in which resuscitation by paramedics was necessary during the defined study period. Since the introduction of the ERC guidelines 2005 and 2010, more applications of the LT-D have taken place than previously (own data of an earlier investigation concerning the use of the LT-D before the new guidelines were established). Concerning the guidelines 2005 , we found about $50 \%$ of all resuscitations (82/150) documented LT-D placements versus $n=42$ (about $48 \%$ of all resuscitations: 42/100) concerning the guidelines 2010 ( $p>0.05)$. However, during resuscitation, a large number of patients during the defined investigation time (about 50\%) had no LT-D inserted at all. These patients were treated by prehospital emergency physicians and/or paramedics, using ETI and BMV ventilation, or BMV ventilation alone (own data).

All patients were adults (age: range: 18-95 years, median: 73 years, mean: 71.3 years, $\mathrm{SD} \pm 14.8$ years; height: range: 150-190 cm, median: $175 \mathrm{~cm}$, mean: $171.2 \mathrm{~cm}, \mathrm{SD} \pm 8.3)$. In line with the manufacturer's instructions, the LT-D sizes used in 29 cases were size $5(22 \%)$, in 92 cases size $4(71 \%)$, and in 9 cases size $3(7 \%)$. All paramedics had more than 2 years' working experience. All of them had practical knowledge in using the LT-D. With regard to practical experiences in using the LT-D, no statistically significant differences between the included paramedics could be seen (according to working experience; $p>0.05$ ). Therefore, all paramedics were described as one group in our results.

\section{Number of attempts to place the LT-D/adequate ventilation}

Overall, $83 \%(n=108)$ of the paramedics placed the LT-D correctly at the first attempt. Successful placement was determined by pulmonary auscultation. More than one attempt was necessary in $n=13$ cases $(10 \%)$. In $n=9(7 \%)$ cases, there was no correct placement of the LT-D and, therefore, no adequate ventilation was determined by pulmonary auscultation. In those situations, paramedics used another airway device (eg, BMV ventilation and/or ETI). Adequate ventilation was possible in $\mathrm{n}=107$ cases $(82.3 \%)$.

\section{Time needed for insertion}

With regard to the ERC guidelines $2005, \mathrm{n}=123$ of all paramedics $(95 \%)$ were able to place the LT-D within the recommended $30 \mathrm{~s}$ for ETI. With regard to the ERC guidelines 2010, $n=57$ of all paramedics (44\%) were able to place the LT-D within the recommended $10 \mathrm{~s}$ (according to the placement of airway devices during resuscitation; $\mathrm{p}<0.001$ ) successfully. Time needed for insertion, and number of placement attempts required is shown in table 1 . There were no statistically significant differences $(p>0.05)$ between participants who worked concerning the ERC guidelines of 2005 versus those to the ERC guidelines of 2010 .

\section{Situation of LT-D placement/insertion during resuscitation}

Placement of the LT-D as a first-line airway device was started initially after having determined unconsciousness and cardiac arrest in $n=95(73 \%)$ of all cases. After using BMV ventilation,
Table 1 Time needed for successful insertion of the laryngeal tube disposable, and number of placement attempts required

\begin{tabular}{llll}
\hline Time (\%) & & & \\
$<10 \mathrm{~s}$ & $10-20 \mathrm{~s}$ & $21-30 \mathrm{~s}$ & $>30 \mathrm{~s}$ \\
$57(43.8)$ & $58(44.6)$ & $8(6.2)$ & $7(5.4)$ \\
Number of placement attempts required (\%) & & \\
1 & $>1$ & & \\
$108(83)$ & $22(17)$ & & \\
\hline
\end{tabular}

the LT-D was placed by $n=35(27 \%)$ during the first $2-4 \mathrm{~min}$ of performing the resuscitation process. In those situations, until the LT-D was placed successfully, paramedics had to ventilate the patient by using BMV ventilation.

\section{Problems/complications}

In $n=29$ of all reported cases (22.3\%), different problems were described by the paramedics. Cuff problems were the most reported. However, in all 10 cases $(7.7 \%)$ in which problems with the cuff pressure were described, paramedics had to use another LT-D. After using the second LT-D, no more cuff problems were subsequently described.

Obvious regurgitation or vomiting was documented in six cases prior to LT-D intubation. There were no known occurrences of regurgitation during or after LT-D placement. Morbid obesity of the patient was reported as another problem in which the use of the LT-D was not successful $(B M I \geq 40)$. Overall, in $\mathrm{n}=23(19.7 \%)$ of all cases, paramedics reported that adequate ventilation was not possible. The problems and complications are shown in table 2 .

\section{Removal of the LT-D}

Table 3 shows where or by whom the LT-D was removed.

\section{Return of spontaneous circulation}

ROSC at the scene, following out-of-hospital resuscitation, was recorded in 37\% (48/130) of all cases. In about 63\%, it was not possible to achieve ROSC. After a time period of $24 \mathrm{~h}$, ROSC was described in $n=8(6.2 \%)$; in about $63 \%(n=84)$ no ROSC were described; in all other cases $(n=38)$ no information about ROSC was required.

\section{DISCUSSION}

The LT-D is recommended as an alternative airway device to BMV and to ETI during resuscitation, especially for those paramedics and prehospital emergency physicians not trained and experienced with ETI continuously. ${ }^{1}{ }^{2}$ The 2005 and 2010 ERC guidelines intended to reduce the HOT during resuscitation as

Table 2 Problems and complications

\begin{tabular}{lrl}
\hline Problem/complication & $\mathbf{n}(\%)$ & No adequate ventilation \\
\hline Cuff pressure problems & $10(7.7)$ & 5 (before using another LT-D) \\
Morbid obesity & $6(4.6)$ & 6 \\
Vomiting/regurgitation & $6(4.6)$ & 6 \\
Fixation was not possible & $3(2.3)$ & 2 \\
Tongue swelling & $3(2.3)$ & 3 \\
Laryngeal spasm & $1(0.8)$ & 1 \\
\hline
\end{tabular}

LT-D, laryngeal tube disposable 
Table 3 Reasons for replacing the laryngeal tube disposable (LT-D)

\begin{tabular}{lc}
\hline Reasons & $\mathbf{n}(\%)$ \\
\hline No removal of the LT-D & $18(13.8)$ \\
Replacement by a prehospital emergency physician & $51(39.2)$ \\
Replacement by a paramedic & $5(3.9)$ \\
Other reasons (eg, death of the patient) & $56(43.1)$ \\
\hline
\end{tabular}

Patients in whom no return of spontaneous circulation during resuscitation was seen were included as well.

much as possible. ${ }^{12} 17$ The use of SADs for airway management may be of special importance for the reduction of the HOT. SADs should not be used only as a rescue device after failed ETI. ${ }^{18}$ Moreover, to further minimise HOT, the time to manage the airway and the time for ventilation by the use of SADs should be reduced. Different simulator-based studies could show that the use of the LT-D, instead of ETI or BMV, reduces the HOT significantly. ${ }^{19-23}$ Moreover, a high incidence of complications, an unacceptably high HOT and a poor success rate when using ETI during cardiac arrest are described in the literature. ${ }^{24-29}$ An easy-to-use and effective alternative airway device to ETI (eg, the LT-D) is therefore necessary. In the investigated EMS system, the LT-D was implemented as an alternative airway device to BMV and ETI in 2008.

The results of the present investigation show that the LT-D was easy to handle in most cases. In $83 \%$ of all reported cases, the LT-D could be placed within one attempt. Moreover, in 93\% of all cases, the LT-D could be placed in two attempts. Our findings are in accordance with previously published results during anaesthesia for operative procedures and from manikin studies. $679102030-32$ However, rates of more than $95 \%$ on the first attempt described in the literature could not be confirmed in the present study. Practical experience in using the LT-D had no statistically significant influence concerning the placement of the LT-D. This corresponded with the study of Schalk et al. ${ }^{9}$ As suggested in earlier studies, it seems to be speculative as to how many ETI placement attempts may be unsuccessful and how many may have a longer insertion time than $30 \mathrm{~s}^{9}$ However, we also have to report that the LT-D placement failed in 7\%; moreover, adequate ventilation was not possible in about $19 \%$ of all cases. These results do not correspond with earlier investigations. ${ }^{8-10}$ Only in a few cases in which the ventilation was possible and successful, the LT-D was used as an airway device in the emergency department as well and was removed later on. Although we did not find an insertion success rate of 100\% like previous manikin studies described, we may underline the use of the LT-D as a first-line SAD during resuscitation, because of its ease of handling, overall high success rate and acceptable HOT. Further investigations were able to show similar results concerning the use of the LT-D during out-of-hospital resuscitation. $^{8-10} 32$ According to recent data, most paramedics were able to place the LT-D within 10-20 s, thus remaining under the $30 \mathrm{~s}$ to accomplish ETI successfully, as recommended by the ERC in 2005, and within the $10 \mathrm{~s}$ in $2010 .^{1}{ }^{2}$ Using the LT-D, airway management could be performed in most cases without discontinuing chest compressions. Taking this fact into account, the LT-D meets the criteria for a short HOT as the ERC suggests. Earlier manikin studies and observational studies in patients demonstrated comparable results concerning significantly reduced HOT. ${ }^{38-10}$
However, one result of our investigation has to be mentioned: several problems and complications due to the insertion and ventilation were mentioned by the paramedics. In particular, problems in patients with morbid obesity (inadequate ventilation in all cases) were described. For this, an alternative airway management (eg, ETI by using video-laryngoscopy) should be discussed as well. We also have to mention that the problems of our investigation cannot be generalised, because it seems to be a single observation from our study. In all described cases, vomiting was observed before the LT-D was placed. The complications in patients with vomiting are also described in other investigations. ${ }^{9}$ Schalk et $a l^{9}$ also described cases in which regurgitation was associated with the use of the LT-D. For those cases, the LTS-D with a gastric drain tube may be a good alternative. With regard to placement success, placement time and HOT, no statistically significant difference between the LT-D and the LTS-D could be shown in a manikin study (own data). By comparison, using BMV ventilation for airway management, complications like regurgitation are described in about $12 \%$ of all cases. ${ }^{33}$ When using a laryngeal mask as an alternative airway device during resuscitation, regurgitation and vomiting are reduced to $2 \% .^{33}$ Taking into account this percentage for SADs, the described number of regurgitation and vomiting incidents seems to be realistic. However, because of this, the authors note that with frequent use of the LT-D during resuscitation, such problems can occur more often. Furthermore, we were not able to evaluate the high unknown numbers for microaspiration and regurgitation.

Normally, the LT-D should be left in place throughout resuscitation and transport to hospital. After ROSC, the LT-D should be removed in hospital by a physician who has experience in performing ETI. In the present study, the LT-D was removed in about $85 \%$ of all cases. Retrospectively, we were not able to find reasons for this. Like earlier studies, we also recommend removal of the LT-D in hospital. ${ }^{9} 10$ Although prehospital emergency physicians are able to remove the LT-D and to use ETI as an airway device, the conditions for intubating a patient by using ETI are better in hospital than in a prehospital setting. ${ }^{27}$ Therefore, an LT-D which enables adequate ventilation should be removed in hospital.

With regard to ROSC, there were similar results to earlier investigations. 12103435 However, the aim of this investigation was not an evaluation of patient outcome after cardiac arrest. According to our described problems and complications, the LT-D had no negative effects with regard to ROSC compared

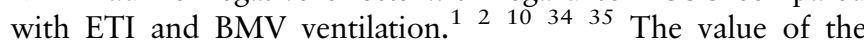
LT-D as a regular alternative airway device concerning patients' outcome requires assessment in further clinical investigations. Furthermore, based on the review by Deakin et $a l^{36}$ 'SAD should be introduced into all ambulance services ...', we recommend that it seems to be necessary to discuss the use of all SADs during resuscitation in a new kind of way to decide the appropriate way in future.

Overall, there are several advantages concerning the use of the LT-D as a SAD. These were mentioned in different manikin studies, in patient studies during surgery and resuscitation, and in documented case reports. However, to sum up, the LT-D seems to represent an acceptable first-line alternative airway device for artificial respiration during resuscitation.

\section{LIMITATIONS}

The present investigation has several limitations. First, the design of the study is an observational one. Therefore, lack of comparison of the investigated LT-D with other alternative 
airway devices, BMV or ETI is one obvious limitation of this study. Second, because of the standardised use of the LT-D during resuscitation within our defined EMS system, we only tested this airway device, assuming that a comparison study was not possible at this time. Third, we were not able to assess all airway management procedures during the defined period. Fourth, the LT-D was only used as an alternative airway device during resuscitation. We are not able to discuss its use during other emergency situations in which ventilation is necessary. Fifth, the LT-D was not used as an alternative airway device in the case of failed ETI. In most cases it was used as a first-line device. This fact may be a positive bias concerning the reported high placement success rate. Finally, we have to mention that ventilation/oxygenation success was not determined by using capnometry or capnography. At the time of our investigation, there was no regularly requirement for paramedics to use $\mathrm{ETCO}_{2}$ to authenticate correct placement of the LT-D in Germany.

\section{CONCLUSIONS}

In the present investigation, paramedics documented their experiences with the use of the LT-D during resuscitation. According to these experiences, the LT-D was a successful alternative SAD overall. The LT-D is an easy-to-handle alternative for airway management during resuscitation, especially for paramedics unfamiliar with ETI. However, we were able to describe more problems in the use of the LT-D than earlier investigations. Therefore, in future, more investigations concerning the use of alternative airway devices in comparison with ETI and/or videolaryngoscopy seem to be necessary. Concerning our study results and those which we can find in the literature, the author group recommends the LT-D as useful SDA. Furthermore, we recommend the use of the LT-D for paramedics who are not familiar in the use of tracheal intubation.

Acknowledgements The authors would like to thank all the paramedics from the participating emergency medical service for their efforts during this investigation. The problems in the use of alternative airway devices are identified only by practice.

Contributors JUM, TFS, CHRW, TS and RS participated in designing the study. JUM, CHRW, TS and RS participated in collecting and entering the data. JUM, AFP and BMG supported in editing the manuscript. BMG cowrote the manuscript and added important comments to the paper. All authors read and approved the final manuscript.

Competing interests TS: Author of manufacturer instructions concerning the use of the LT-D (VBM Medizintechnik Sulz a. N., Germany). This work is based on an investigation which was published by the author group in 'Resuscitation' in 2009. This work also contains data from a doctoral thesis (JUM). This research received no specific grant from any funding agency in the public, commercial, or not-for-profit sectors.

Provenance and peer review Not commissioned; externally peer reviewed.

\section{REFERENCES}

1 Deakin CD, Nolan JP, Soar J, et al. European resuscitation council guidelines for resuscitation 2010 section 4 . Adult advanced life support. Resuscitation 2010;81:1305-52.

2 European Resuscitation Council. European resuscitation council guidelines for resuscitation 2005. Resuscitation 2005;67(S1):S1-89.

3 Ruetzler K, Gruber C, Nabecker S, et al. Hands-off time during insertion of six airway devices during cardiopulmonary resuscitation: a randomised manikin trial. Resuscitation 2011;82:1060-3.

4 Gaitini L, Madrid V, Capdevila M, et al. The laryngeal tube. Rev Esp Anestesiol Reanim 2008;55:232-41.

5 Asai T, Shingu K. The laryngeal tube. Br J Anaesth 2005;95:729-36.

6 Dörges V, Ocker H, Wenzel V. The laryngeal tube: a new simple airway device. Anaesth Analg 2000;90:1220-2.
7 Agro F, Cataldo R, Alfano A, et al. A new prototype for airway management in an emergency: the laryngeal tube. Resuscitation 1999;41:284-6.

8 Heuer JF, Barwing J, Eich C, et al. Initial ventilation through laryngeal tube instead of face mask in out-of-hospital cardiopulmonary arrest is effective and safe. Eur J Emerg Med 17:10-15.

9 Schalk R, Byhahn C, Fausel F, et al. Out-of-hospital airway management by paramedics and emergency physicians using laryngeal tubes. Resuscitation 2010;81:323-6.

10 Wiese $\mathrm{CH}$, Semmel T, Müller JU, et al. The use of the laryngeal tube disposable (LT-D) by paramedics during out-of-hospital resuscitation-an observational study concerning ERC guidelines 2005. Resuscitation 2009;80:194-8.

11 Asai T, Kawashima A, Hidaka I. Use of the laryngeal tube in patients without teeth. Resuscitation 2001;51:213-14.

12 Asai T, Shingu K, Cook T. Use of the laryngeal tube in 100 patients. Acta Anaesthesiol Scand 2003;47:828-32.

13 Ocker $\mathrm{H}$, Semmel T. The laryngeal tube in emergency medicine: a practical approach to its use. Medakademie Publishers 2007;1:1-19 ISBN 978-3-00-0109207-8.

14 Wik L, Kramer-Johansen J, Myklebust $\mathrm{H}$, et al. Quality of cardiopulmonary resuscitation during out-of-hospital cardiac arrest. JAMA 2005;293:299-304.

15 World Medical Association. World Medical Association Declaration of Helsinki. Ethical principles for medical research involving human subjects. Nurs Ethics 2002;9:105-9.

16 Ludbrook J. On making multiple comparisons in clinical and experimental pharmacology and physiology. Clin Exp Pharmacol Physiol 1991:379-92.

17 Jäntti $H$, Kuisma M, Uusaro A. The effects of changes to the ERC resuscitation guidelines on no flow time and cardiopulmonary resuscitation quality: a randomised controlled study on manikins. Resuscitation 2007;75:338-44.

18 Cobas M, De la Pena MA, Manning A, et al. Prehospital intubations and mortality: a level one trauma center perspective. Anesth Analg 2009;109:489-93.

19 Genzwuerker HV, Finteis T, Slabshi D, et al. Assessment of the use of the laryngeal tube for cardiopulmonary resuscitation in a manikin. Resuscitation 2001;51:291-4.

20 Genzwuerker HV, Oberkinkhaus J, Finteis T, et al. Emergency airway management by first responders with the laryngeal tube-intuitive and repetive use in a manikin. Scand J Trauma Resusc Emerg Med 2005;13:1-4.

21 Wiese CHR, Bahr J, Bergmann A, et al. Reduction in no flow time using a laryngeal tube: comparison to bag-mask ventilation. Anaesthesist 2008;57:589-96.

22 Wiese CHR, Bartels U, Bergmann A, et al. Using a laryngeal tube during cardiac arrest reduces 'no flow time' in a manikin study: a comparison between laryngeal tube and endotracheal tube. Wien Klin Wochenschr 2008;120:217-23.

23 Wiese CHR, Bartels U, Schultens A, et al. Influence of airway management strategy on no-flow-time during an advanced life support course for intensive care nursesa single rescuer resuscitation manikin study. BMC Emerg Med 2008;8:4.

24 Bradley JS, Billows GL, Olinger ML, et al. Prehospital oral endotracheal intubation by rural basic emergency medical technicians. Ann Emerg Med 1998;32:26-32.

25 Reinhart DJ, Simmons G. Comparison of placement of the laryngeal mask airway with endotracheal tube by paramedics and respiratory therapists. Ann Emerg Med 1994;24:260-3.

26 Sayre M, Sackles JC, Mistler AF, et al. Field trial of endotracheal intubation by basics EMTs. Ann Emerg Med 1998;32:228-33.

27 Timmermann A, Braun U, Panzer W, et al. The out-of-hospital esophageal and endobronchial intubations performed by emergency physicians. Anaesth Analg 2007;104:619-23.

28 Katz FH, Falk JL. Misplaced endotracheal tubes by paramedics in an urban emergency medical service system. Ann Emerg Med 2001;37:62-4.

29 Ochs M, Davis D, Hoyt D, et al. Paramedic-performed rapid sequence intubation of patients with severe head injuries. Ann Emerg Med 2002;40:168-71.

30 Genzwuerker HV, Dhonau S, Ellinger K. Use of the laryngeal tube for out-of-hospital resuscitation. Resuscitation 2002;52:221-4.

31 Kette F, Reffo I, Giordani G, et al. The use laryngeal tube by nurses in out-of-hospital emergencies: preliminary experience. Resuscitation 2005;66:21-5.

32 Yildiz TS, Solak M, Toker K. Comparison of laryngeal tube with laryngeal mask airway in anaesthetized and paralysed patients. Eur J Anaesthesiol 2007;24:620-5.

33 Baskett PJF. The use of the laryngeal mask airway by nurses during cardiopulmonary resuscitation. Results of a multicentre trial. Anaesthesia 1994;49:3-7.

34 Ishtiaq 0, Iqbal M, Zubair M, et al. Outcome of cardiopulmonary resuscitationpredictors of survival. J Coll Physicians Surg Pak 2008;18:3-7.

$35 \mathrm{Ma} \mathrm{MH}$, Chiang WC, Ko WC, et al. Outcomes from out-of-hospital cardiac arrest in Metropolitan Taipei: does an advanced life support service make a difference? Resuscitation 2007;74:461-9.

36 Deakin CD, Clarke T, Nolan J, et al. A critical reassessment of ambulance service airway management in prehospital care: Joint Royal Colleges Ambulance Liaison Committee Airway Working Group, June 2008. Emerg Med J 2010;27:226-33. 
The use of the laryngeal tube disposable by paramedics during out-of-hospital cardiac arrest: a prospectively observational study (2008-2012)

Jens-Uwe Müller, Thomas Semmel, Roland Stepan, Timo F Seyfried, Aron F Popov, Bernhard M Graf and Christoph H R Wiese

Emerg Med J 2013 30: 1012-1016 originally published online January 10, 2013

doi: 10.1136/emermed-2012-201923

Updated information and services can be found at:

http://emj.bmj.com/content/30/12/1012

These include:

References This article cites 34 articles, 1 of which you can access for free at: http://emj.bmj.com/content/30/12/1012\#BIBL

Email alerting Receive free email alerts when new articles cite this article. Sign up in the service box at the top right corner of the online article.

Topic Articles on similar topics can be found in the following collections Collections

\section{Notes}

To request permissions go to:

http://group.bmj.com/group/rights-licensing/permissions

To order reprints go to:

http://journals.bmj.com/cgi/reprintform

To subscribe to BMJ go to:

http://group.bmj.com/subscribe/ 